\title{
A Critical Review of Properties and Analytical Methods for the Determination of Docetaxel in Biological and Pharmaceutical Matrices
}

Gilmar Hanck da Silva, Mariza Aires Fernandes, Lucas Noboru Fatori Trevizan, Felipe Tita de Lima, Josimar O. Eloy \& Marlus Chorilli

To cite this article: Gilmar Hanck da Silva, Mariza Aires Fernandes, Lucas Noboru Fatori Trevizan, Felipe Tita de Lima, Josimar O. Eloy \& Marlus Chorilli (2018) A Critical Review of Properties and Analytical Methods for the Determination of Docetaxel in Biological and Pharmaceutical Matrices, Critical Reviews in Analytical Chemistry, 48:6, 517-527, DOI: 10.1080/10408347.2018.1456315

To link to this article: https://doi.org/10.1080/10408347.2018.1456315

曲 Published online: 27 Apr 2018.

Submit your article to this journal ๘

Џلll Article views: 190 


\title{
A Critical Review of Properties and Analytical Methods for the Determination of Docetaxel in Biological and Pharmaceutical Matrices
}

\author{
Gilmar Hanck da Silva (D), Mariza Aires Fernandes (D), Lucas Noboru Fatori Trevizan (D), Felipe Tita de Lima (D), \\ Josimar O. Eloy, and Marlus Chorilli (D)
}

São Paulo State University (UNESP), School of Pharmaceutical Sciences, Araraquara, São Paulo, Brazil

\begin{abstract}
Docetaxel (DTX) is an antineoplastic agent of the second generation of the taxoid family. It is a semisynthetic drug prepared from a precursor extracted of the plant Taxus baccata. The commercial formulation of DTX, Taxotere ${ }^{\circledR}$, employs the surfactant polysorbate 80 , due to the low water solubility of the drug, causing several side effects. Therefore, there is a need to develop delivery systems to reduce the side effects of DTX. In addition, this drug has been qualitative and quantitatively analyzed in pharmaceutical formulations and biological samples. Thus, several techniques and analytical methods have been reported with the aim of optimizing the analytical signal, increasing sensitivity, selectivity and reducing the effects of interference. Herein, we highlight immunoassay, capillary electrophoresis and chromatographic methods. This review presents a summary of physicochemical and pharmacokinetics properties, mechanisms of action, drug delivery systems and analytical methods used in quantification of DTX in diverse matrices such as blood, plasma, oral fluid, urine, carcinoma cells, pharmaceutical formulations and delivery systems.
\end{abstract}

\section{KEYWORDS}

Docetaxel; physicochemical properties; drug delivery systems; analytical methods

\section{Introduction}

Docetaxel (DTX) is an antineoplastic drug of the taxoid family, obtained semi-synthetically from the natural compound 10deacetylbaccarin III, a non-cytotoxic component of the Yew tree, Taxus baccata. ${ }^{[1-3]}$ This drug is highly lipophilic and practically insoluble in water, which is a factor that compromises the pharmacokinetics profile. The commercial formulation, Taxotere $^{\circledR}$, employs the surfactant polysorbate 80 and ethanol to improve the solubility, although causing several side effects. $^{[3-5]}$

The mechanism of action of DTX is related to the cytotoxic activity, consequence of $\beta$-tubulin binding, which causes inhibition of cell proliferation and mitotic progression due to inhibition of microtubule dynamics. ${ }^{[6]}$ DTX was approved by the Food and Drug Administration (FDA) in 1996 for the treatment of anthracycline-refractory metastatic breast cancer. Afterwards, it was approved for treatment of platinum-refractory stage non-small-cell lung cancer. ${ }^{[7,8]}$ Furthermore, the toxicity of both drug and formulations has stimulated the development of nanostructured delivery systems, with many advantages, including controlled DTX release and improved pharmacokinetics. ${ }^{[9,10]}$

The development and validation of analytical methods play an important role both on the research of DTX delivery systems and quality control. Many analytical methods for quantification of DTX have been published, particularly employing high-performance liquid chromatography (HPLC). Overall, those studies reported differences in the parameters of wavelength, mobile phase, stationary phase, and others, which will be addressed herein. ${ }^{[3,11,12]}$ Moreover, other analytical methods reported for DTX quantification include immunoassay, ${ }^{[13,14]}$ capillary electrophoresis (CE) ${ }^{[15]}$ and ultraperformance liquid chromatography (UPLC), which presents many advantages, including the faster analytical run and the use of smaller volumes of solvents. ${ }^{[16-18]}$ Another important issue regarding DTX analytical methods is the analytical matrix, which should not interfere in drug quantification. In this context, several studies have quantified DTX extracted from different matrices, including biological samples, such as blood, plasma, urine, and carcinoma cells ${ }^{[19-22]}$ and also in drug delivery systems. ${ }^{[23-25]}$

In this review, our purpose is to present important physicochemical characteristics of DTX and also give the reader an overview of the drug's pharmacokinetics, the mechanism of action and summarize some strategies to load DTX into nanostructured delivery systems, highlighting the advantages. Finally, we will address the most commonly employed analytical methods for DTX quantification in various matrices, including formulations and biological samples, highlighting the analytical conditions employed.

\section{Physicochemical and pharmacokinetics characteristics}

DTX (CAS number: 114977-28-5, Figure 1) is a white to offwhite crystalline powder with a molecular weight and formula corresponding to $807.89 \mathrm{Da}$ and $\mathrm{C}_{43} \mathrm{H}_{53} \mathrm{NO}_{14}$, respectively. Its solubility in water is $1.27 .10^{-2} \mathrm{~g} / \mathrm{L}$ and melting point is $232^{\circ} \mathrm{C}$.

CONTACT Marlus Chorilli $\otimes$ chorilli@fcfar.unesp.br E São Paulo State University (UNESP), School of Pharmaceutical Sciences, 14800-903, Araraquara, São Paulo Brazil.

(c) 2018 Taylor \& Francis Group, LLC 


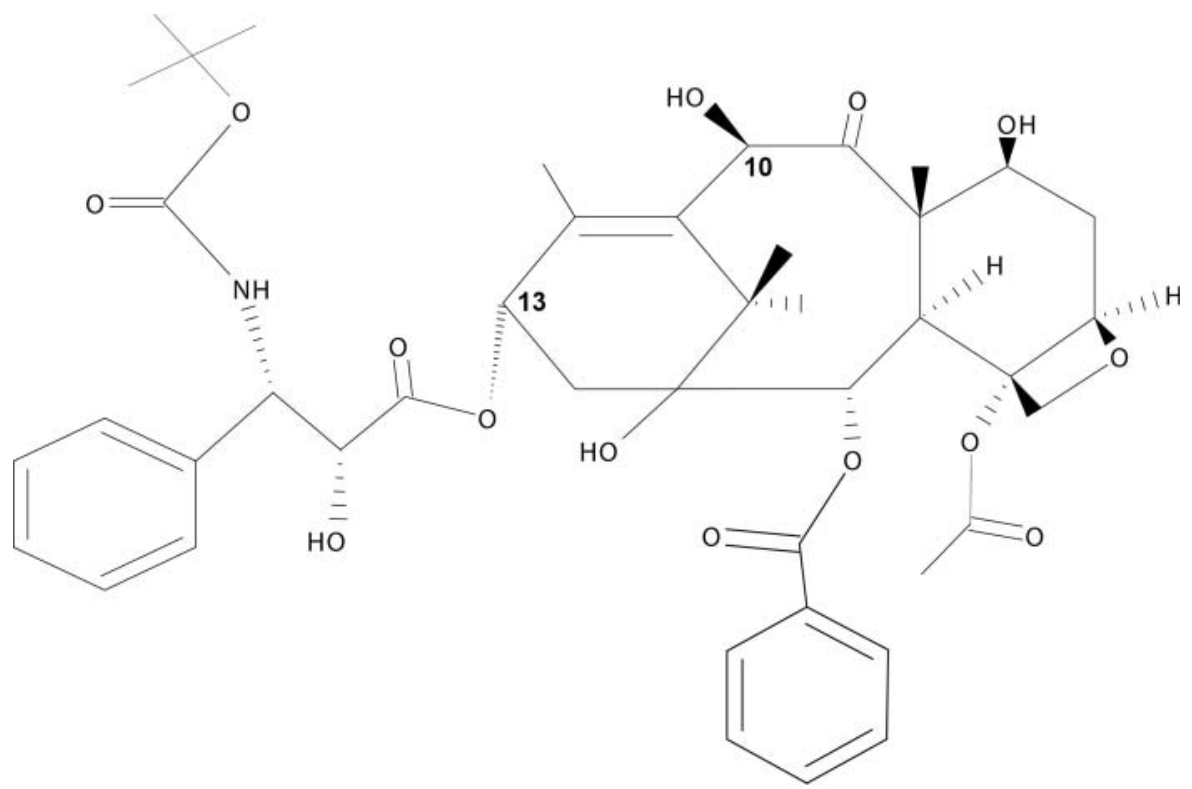

Figure 1. Chemical structure of docetaxel.

DTX is a highly lipophilic, semi-synthetically derived from 10deacetylbaccatin III compound. But, compared to paclitaxel (PTX), it is quickly absorbed, it is less toxic and the water solubility is better, due to differences in their chemical structures at two positions, the C-13 tert-butoxy group instead of the benzamide phenyl group and the $\mathrm{C}-10$ hydroxy group replacing the acetyl group. ${ }^{[26-28]}$

The pharmacokinetic profile of DTX is well characterized, composed of three compartments, with half-lives of $4.5 \mathrm{~min}$, $38.3 \mathrm{~min}$, and $12.2 \mathrm{~h}$ for the alpha, beta, and gamma phases, respectively. ${ }^{[26]}$ The standard dose varies according to the type of cancer and the treatment used, however the recommended dose is between 75 and $100 \mathrm{mg} / \mathrm{m}^{2}$ with infusion once every 3 weeks for $1 \mathrm{~h}$, unlike PTX whose recommended dose varies from 200 to $250 \mathrm{mg} / \mathrm{m}^{2}$. 29,30$]$

The distribution of DTX occurs from the central compartments to the peripheral compartments. The total volume of distribution in the human body is approximately $22 \mathrm{~L} / \mathrm{h} / \mathrm{m}^{2}$ and the mean stationary distribution volume is $113 \mathrm{~L},{ }^{[31]}$ depending on the liver function, age, body surface area, and plasma proteins (for example, albumin, $\alpha$-1-acid glycoprotein e lipoproteins). ${ }^{[32]}$

Previous studies reported DTX binding to plasma proteins, mainly albumin, $\alpha$-1-acid glycoprotein and lipoproteins, about $90-95 \%$, in vitro, and 70-95\%, in vivo. $\alpha$-1-acid glycoprotein is present in high levels in individuals with pathologies such as cancer and therefore becomes the major factor responsible for the total clearance of DTX. In addition, DTX bound to plasma proteins accounts for a $19 \%$ decrease in drug clearance. ${ }^{[28,33]}$

The tissue distribution of DTX is influenced by the route of administration and some authors consider the intravenous route as the best alternative. Preclinical studies in animals have demonstrated high concentrations in most tissues. The highest concentrations were initially found in the liver, stomach, bile ducts, pancreas, muscles, and hematopoietic tissues and after the initial period of administration, absorption by the nervous system and testes is limited. Compared with other tissues, tumors have elevated levels of DTX due to drug binding to the $\alpha$-1-acid glycoprotein. ${ }^{[6,8,14]}$

The cytochrome P450 (CYP3A4 and CYP3A5) system plays an important role on the clearance and metabolism of the drug, resulting in various pharmacologically inactive oxidation products. $^{[28,34,35]}$ After the oxidative metabolism of the tert-butyl ester group, excretion occurs via the biliary, intestinal, and renal organs. At a time interval of 7 days, the urinary and fecal excretion is approximately $6 \%$ and $75 \%$ of the amount administered, respectively. The highest elimination rate occurs during the first $48 \mathrm{~h}$ through feces. ${ }^{[33,36]}$

\section{Mechanisms of action}

DTX acts on microtubules, which are hollow cylindrical fibrillar structures with an outer diameter of approximately $25 \mathrm{~nm}$ and a wall of about $5 \mathrm{~nm}$ thickness composed of $\alpha$-tubulin and $\beta$-tubulin heterodimers firmly attached together by noncovalent bonds. ${ }^{[37,38]}$ They are essential in cellular processes, such as transport, signaling and mitosis, in higher eukaryotic cells, and play an important role on the position of organelles and are responsible for removing the chromosomes in mitosis. ${ }^{[39]}$

Dynamic instability is one of the phenomena caused by microtubules through the loss or addition of heterodimers of alpha or beta tubulin from extremities. In cells, all microtubules originate from a microtubule organizing center. ${ }^{[40]}$ Polymerization of tubulin dimers can be influenced by a number of factors, such as guanosine triphosphate (GTP), which binds to an exchangeable site on $\beta$-tubulin and a non-exchangeable site on $\alpha$-tubulin and microtubule associated proteins (MAPs), which constitute a family of complex proteins responsible for the regulation of the polymerization and function of tubulin. ${ }^{[39,41]}$

The antimitotic agents such as colchicine, podophyllotoxin, and vinblastine have a locking function in the assembly of microtubules. However, DTX, which also acts on the cell cycle, has the opposite effect, provoking the stabilization of the microtubules (Figure 2). ${ }^{[42,43]}$ DTX initially inhibits the 

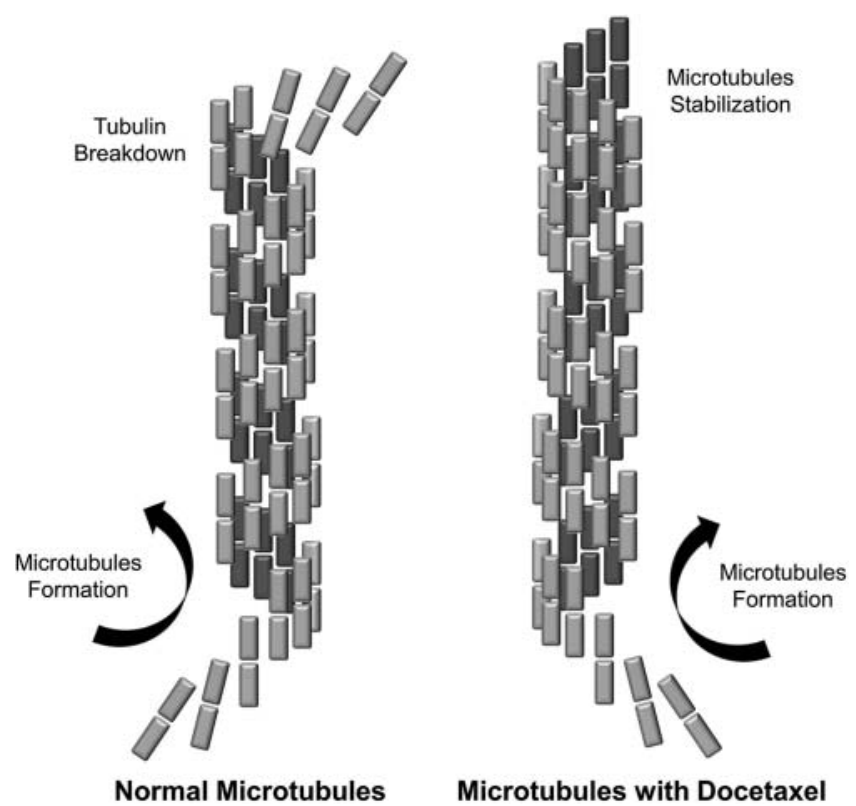

Figure 2. Effect of docetaxel on microtubules. Source: adapted from [45].

disassembly (disruption of microtubules) of the physiological microtubules by binding the $\beta$-tubulin subunit promoting the production of stable microtubule bundles and without normal function. ${ }^{[9,44]}$

The in vitro effects observed in tubulin polymerization by microtubule stabilizing agents (MSA) and DTX are similar to antiproliferative effects at the cellular level. ${ }^{[38]}$ The MSAs and DTX suppress microtubule dynamics and stabilization, causing the interruption of mitosis by cell accumulation in G2/M phase of the cell cycle and subsequent blocking cell division and proliferation of tumor cells. ${ }^{[37,38]}$

As described in the literature, DTX is responsible for regulating microtubules upon binding to tubulin. Its action also involves other intracellular processes, such as binding to proteins of the Bcl2 family, causing phosphorylation, and inactivation and later apoptosis. Some authors described that DTX induces the expression of inhibitors of cyclin - cyclin-dependent kinase (CDK) complexes that cause cell cycle arrest at different stages of mitosis (p21, p27, and p53). Comparatively, DTX has some advantages when compared to PTX, for example, in tumor treatment it is more effective by binding $\beta$-tubulin with greater affinity, causing direct antitumor activity through the induction of apoptosis by phosphorylation of Bcl2. Furthermore, DTS has a longer retention time in tumor cells than PTX due to its structural characteristics. Thus, the various effects and mechanisms associated with DTX, such as regulation of microtubules, apoptosis, and action on genes related to the cell cycle, make it possible to treat various types of tumors. ${ }^{[9,26,37,41,46,47]}$

\section{Drug delivery systems}

DTX can be loaded into numerous nanostructured delivery systems, including liposomes, polymeric nanoparticles, lipid nanoparticles, micelles, dendrimers, and nanoemulsions. ${ }^{[9]}$ Those systems offer several advantages over standard formulations, including better stability, the sustained drug release, improvement of the therapeutic effect of the drug and better pharmacokinetic profile, with longer circulation times. ${ }^{[10,48,49]}$ Furthermore, nanostructured systems are used in the cancer therapy, since they promote the passive targeting of the drug to the tumor, through the enhanced permeability and retention (EPR) effect. ${ }^{[50]}$

Among the nanocarriers, liposomes, vesicular lipid systems, are the most commonly studied, owing to their biodegradability and low toxicity, compared to other nanocarriers such as nanoemulsions, dendrimers, liposomes, polymeric nanoparticles, and micelles. ${ }^{[51]}$ Several liposomal characteristics are important for DTX delivery and the composition plays a central role, which is demonstrated in several reports in the literature. ${ }^{[52-55]}$ For instance, Pereira et al. ${ }^{[56]}$ evaluated the effect of different lipids, DOPC, DPPC, and DSPC, on both the purification of DTX-loaded liposomes and cytotoxicity on prostate cancer (PC3) spheroids. The results showed that the use of DOPC, the only unsaturated lipid, caused higher loading compared to more rigid liposomes, prepared with the saturated lipids DPPC and DSPC. Furthermore, DSPE-PEG2000 was also used in all formulations, since PEGylation increases the half-life of the liposome in systemic circulation and reduces the macrophage recognition and immunogenicity. The in vitro toxicity of the purified and non-purified liposomes was also determined, showing the importance of the purified liposomes effect on the therapeutic efficacy, since lipid composition improved DTX solubility and tissue penetration.

An alternative to the use of liposomes for DTX delivery is represented by solid lipid nanoparticles (SLN) and nanostructured lipid carriers (NLC), which present improved colloidal stability compared to liposomes, because SLN are composed of a solid matrix, while NLC can be made with both solid and liquid lipids. ${ }^{[57]}$ Liu et al. ${ }^{[58]}$ designed a DTX-loaded nanostructured lipid carrier (DTX-NLC) with the aim of increasing drug therapeutic effect, compared to a regular DTX formulation, Duopafei ${ }^{\circledR}$. The in vitro cytotoxicity was determined using human hepatocellular liver carcinoma (HepG2), ovary cancer cells (SKOV3), lung adenocarcinoma (A549), while the in vivo study was done with murine malignant melanoma (B16). Their results showed that DTX can be well loaded into the NLC, due to its physicochemical properties and lipid composition. In comparison to Duopafei ${ }^{\circledR}$, the DTX-NLC formulation induced more apoptosis in vitro and had superior anticancer efficacy in vivo.

The biodegradable and biocompatible properties of phosphatidylethanolamine (PE) are important characteristics for its use in some nanocarriers. For instance, Zhang et al. ${ }^{[59]}$ prepared DTX loaded-lipid nanoparticles (DTX-LPNPs), based on PE. Their aim was to compare DTX-LPNPs with DTX-loaded nanoparticles (DTX-NPs), composed of a single chemical emulsifier, polyvinyl alcohol (PVA), instead of PE, and the commercial formulation, Duopafei ${ }^{\circledR}$. Their results demonstrated that DTX was well-incorporated in the emulsified system, based on PE, causing better in vitro antitumor effect than DTX-NPs and Duopafei ${ }^{\circledR}$.

Another potential nanostructure for drug delivery is represented by polymeric micelles, which are composed of amphiphilic copolymers. Raza et al. ${ }^{[60]}$ explored the potential of a new DTX-loaded polymeric micelles, composed of tethered poly(lactic-co-glycolic acid) (PLGA) and dextran, a 
biocompatible polysaccharide, which was chemically linked to PLGA, with advantages, such as immuno-neutrality and better pharmacokinetic profile. The in vitro study on MCF-7 and MDA-MB-231 cell lines demonstrated enhanced cytotoxicity of approximately $100 \%$, in both cell cultures. Moreover, the pharmacokinetic profile study, evaluated in rats, demonstrated an increase in the biological half-life and bioavailability, by approximately 5 times compared to the pure drug.

Currently, aside from the passive targeting, there are numerous ways to offer active targeting. One of the most common strategies employ surface modification with folic acid (FA), peptides, dendrimers, and monoclonal antibodies. ${ }^{[61]}$ This strategy is commonly reported to improve efficacy and reduce drug side effects. However, there are few reports regarding surface functionalization on DTX-loaded nanoparticles. For instance, Nateghian et al. ${ }^{[62]}$ conjugated FA or biotin onto DTX-loaded albumin nanoparticles for active targeting to cancer cell lines that overexpress FA or biotin receptors, while Raju et al. ${ }^{[63]}$ prepared D- $\alpha$-tocopheryl polyethylene glycol 1,000 succinate (TPGS) loaded liposomes conjugated with trastuzumab for controlled and targeted delivery of DTX to cancer cells. Kulhari et al. ${ }^{[64]}$ developed both DTX-loaded nanoparticles (DNP) and bombesin peptide (BBN) conjugated DTXloaded nanoparticles (BDNP) for targeted delivery to MDAMB-231 (breast cancer) cell line, which overexpresses gastrinreleasing peptide (GRP) receptor, for comparison with free DTX and the commercial formulation, Taxotere ${ }^{\circledR}$. Their results demonstrated that DNP and, especially, BDNP had more in vitro cytotoxic effect than Taxotere ${ }^{\circledR}$ and free DTX, showing great potential for DTX delivery to GRP receptor-positive cancer cells.

\section{Analytical methods}

Much progress has been made in the last years in the development and validation of analytical methods for identification and quantification of drugs and other molecules of interest in different matrices. However, there is an increasing need to optimize many of these methods, in order to improve the analytical signal, reduce the analysis time, and consequently the consumption of reagents and solvents. ${ }^{[65,66]}$

Several analytical methods were developed for analysis of DTX, with the purpose of identification and quantification in biological samples (Table 1) and also in diverse matrices (Table 2), such as delivery systems, that were presented in the previous section of this review. These methods include, mostly, immunoassays, ${ }^{[13]} \mathrm{CE}$ assays, ${ }^{[15]}$ and chromatographic assays.

The immunoassay methods consist of a competitive reaction using selective anti-DTX monoclonal antibodies-coated nanoparticles followed by the agglutination quantification reaction measured by the absorbance at $660 \mathrm{~nm} .{ }^{[13,14]}$ The technique was compared with LC-MS/MS for determination of the DTX concentration in human plasma by Geng et al. ${ }^{[14]}$ The separation of DTX from plasma by LC-MS/MS utilized a $\mathrm{C}_{18}$ Diamonsil column $(150 \mathrm{~mm} \times 4.6 \mathrm{~mm}$ i.d. $5.0 \mu \mathrm{m}$ particle size $)$ with a mobile phase composed of $0.1 \%$ formic acid:acetonitrile $(\mathrm{ACN})(40: 60, \mathrm{v} / \mathrm{v})$ and the mass spectrometry (MS) was performed in the positive ion multiple reaction monitoring
(MRM) mode, with an ion transition of $\mathrm{m} / z$ 830.5 $>550.4$, resulting in a retention time at $7.5 \mathrm{~min}$. The immunoassay method was carried out according to the protocol presented by Cline et al. ${ }^{[13]}$ and using the DTX reagent kit (Saladax MyDocetaxel assay reagent kit). The absorbance was determined by the difference between the reaction at cycle 27 and cycle 13 without subtraction of a blank reagent. Both methods were validated in accordance with FDA guidelines, including the assessment of specificity, matrix effect, recovery, linearity, accuracy, precision, and stability. However, the immunoassay method was regarded as far more cost-effective and had a better outcome in clinical drug monitoring.

CE is a separation technique based on the electrophoretic mobility of charged species (analytes) under an electric field in a capillary. CE is also used as a synonym of capillary zone electrophoresis (CZE), but there are other electromigration techniques such as micellar electrokinetic chromatography (MEKC), microemulsion electrokinetic chromatography (MEEKC), among others. The technique exhibits numerous advantages: (1) it can use a high voltage (up to $800 \mathrm{~V} / \mathrm{cm}$ ) due to the physical properties of fused silica capillaries; (2) requires small amounts of samples and buffer due to the small dimension of the capillaries; (3) contains a high number of theoretical plate $(N)>10,000$, which contribute to the high efficiency of separation; and (4) it can contain additives which interact with the analytes, altering their electrophoretic mobility and therefore improving separation process. ${ }^{[67]}$

Shakalisava et al. ${ }^{[15]}$ demonstrated the versatility of $\mathrm{CE}$ methods (CZE, MEKC, and MEEKC) for the separation of different types of anticancer drugs, anthracyclines (doxorubicin, daunorubicin, and epirubibin) and taxanes (PTX and DTX) simultaneously. Separation conditions employed UV detection at $230 \mathrm{~nm}$, capillary of $56 \mathrm{~cm}(48 \mathrm{~cm}), 50 \mathrm{~mm}$ i.d., $20^{\circ} \mathrm{C}$. The mobile phases were different according to the technique. For CZE, it corresponded to $20 \mathrm{mM} \mathrm{pH} 9$ borate buffer, $70 \% \mathrm{ACN}$, $30 \mathrm{kV}$, with hydrodynamic injection of $6 \mathrm{~s}$; while for MEKC it was composed of $25 \mathrm{mM} \mathrm{pH} 9$ Tris-phosphate buffer, $70 \%$ methanol, $100 \%$ sodium dodecyl sulfate (SDS), $30 \mathrm{kV}$, with hydrodynamic injection of $12 \mathrm{~s}$; and for MEEKC the mobile phase corresponded to $20 \mathrm{mM} \mathrm{pH} 2.5$ phosphate buffer, $0.81 \%$ $\mathrm{w} / \mathrm{w}$ octane, $3.31 \% \mathrm{w} / \mathrm{w}$ SDS, and $6.61 \% \mathrm{w} / \mathrm{w}$ butan-1-ol, $25 \%$ $\mathrm{ACN},-25 \mathrm{kV}$, with hydrodynamic injection of $12 \mathrm{~s}$. In conclusion, the methods showed high-speed, especially the MEEKC method, with a migration time less than $3 \mathrm{~min}$ and great potential for simultaneous monitoring of pharmacokinetics of anthracyclines and taxanes in plasma.

Among the most studied analytical methods, HPLC methods play a central role, with a variety of detectors, including the ultraviolet (UV) (225-232 nm), most commonly used, MS or tandem mass spectrometry (MS/MS), due to its higher sensitivity, resolution, and possibility to work with different matrices, as well as eliminating interference. HPLC technique is the standard method of the United States Pharmacopoeia (USP 35nd), which uses as mobile phase a mixture of water-ACN and UV detection at $232 \mathrm{~nm} \cdot{ }^{[68]}$ In addition to HPLC, more recently, methods using UPLC with MS or MS/MS as detectors (Table 3) have received great attention, employing particles smaller than $2 \mu \mathrm{m}$ in diameter to achieve superior resolution, speed, and sensitivity compared with HPLC. ${ }^{[69]}$ 
Table 1. HPLC analysis performed with biological samples.

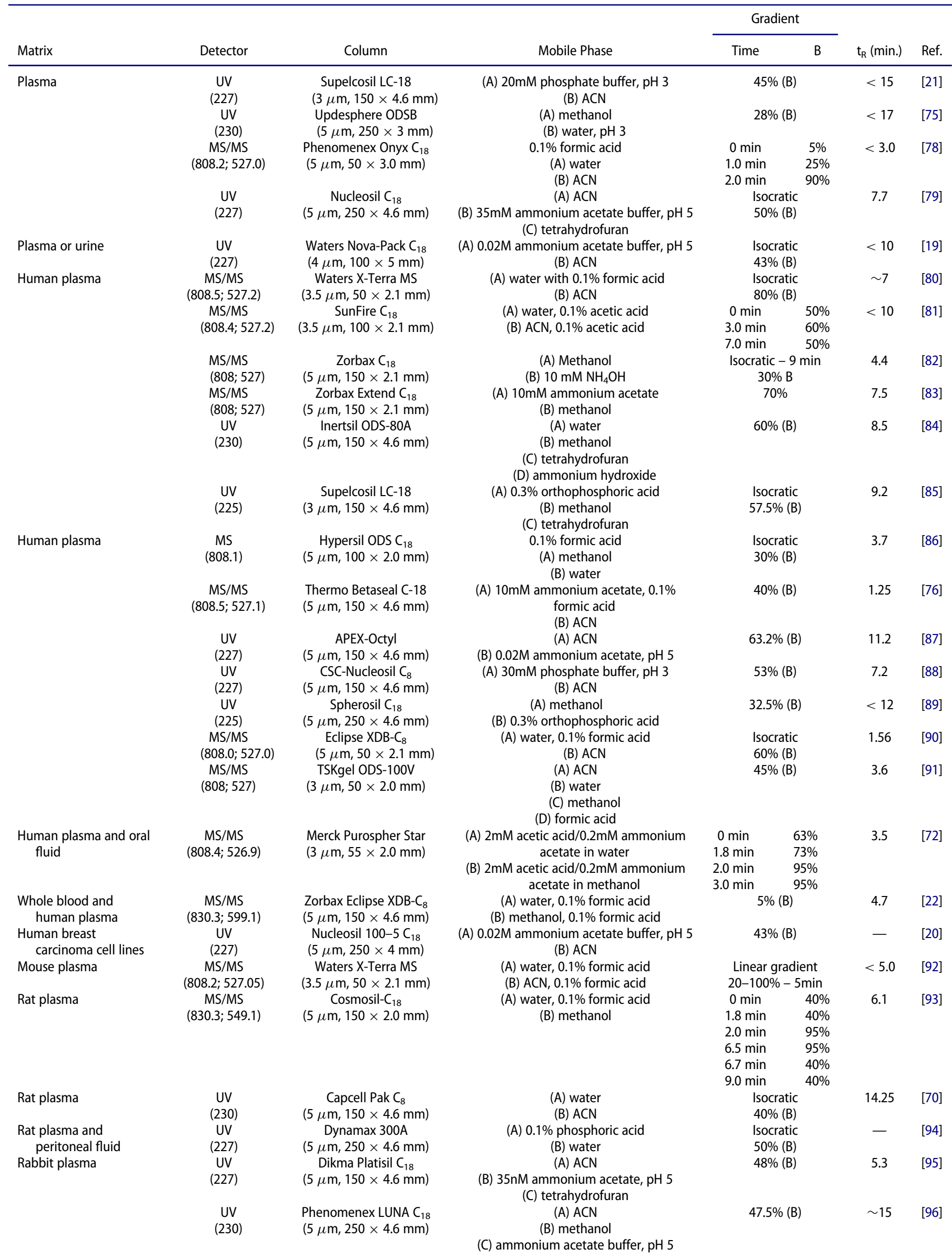


Table 2. HPLC analysis performed with diverse matrices.

\begin{tabular}{|c|c|c|c|c|c|c|c|}
\hline \multirow[b]{2}{*}{ Matrix } & \multirow[b]{2}{*}{ Detector } & \multirow[b]{2}{*}{ Column } & \multirow[b]{2}{*}{ Mobile Phase } & \multicolumn{2}{|c|}{ Gradient } & \multirow[b]{2}{*}{$t_{R}$ (min.) } & \multirow[b]{2}{*}{ Ref. } \\
\hline & & & & Time & B & & \\
\hline Docetaxel pure drug & $\begin{array}{l}\text { UV } \\
(225)\end{array}$ & $\begin{array}{c}\text { Devenosil } C_{18} \\
(5 \mu \mathrm{m}, 150 \times 4.6 \mathrm{~mm})\end{array}$ & $\begin{array}{l}\text { (A) phosphate buffer, pH } 4.2 \\
\text { (B) ACN }\end{array}$ & \multicolumn{2}{|c|}{$\begin{array}{l}\text { Isocratic } \\
70 \%(B)\end{array}$} & 3.19 & [97] \\
\hline $\begin{array}{l}\text { Docetaxel pure drug } \\
\text { and formulation }\end{array}$ & $\begin{array}{l}\text { UV } \\
(230)\end{array}$ & $\begin{array}{c}\text { YMC pack } \\
\text { ODS-A C } 18 \\
(5 \mu \mathrm{m}, 150 \times 3.9 \mathrm{~mm})\end{array}$ & $\begin{array}{c}\text { (A) 60:40 water:ACN } \\
\text { (B) ACN }\end{array}$ & $\begin{array}{c}0 \mathrm{~min} \\
26 \mathrm{~min} \\
66 \mathrm{~min} \\
67 \mathrm{~min} \\
71 \mathrm{~min} \\
72 \mathrm{~min}\end{array}$ & $\begin{array}{c}0 \% \\
0 \% \\
83 \% \\
100 \% \\
100 \% \\
0 \%\end{array}$ & $\sim 21$ & [98] \\
\hline $\begin{array}{l}\text { Docetaxel in parenteral } \\
\text { dosage form }\end{array}$ & $\begin{array}{l}\text { UV } \\
(230)\end{array}$ & $\begin{array}{c}\text { Unison } \mathrm{YMC} \mathrm{C}_{18} \\
(5 \mu \mathrm{m}, 250 \times 4.6 \mathrm{~mm})\end{array}$ & $\begin{array}{c}\text { (A) } 0.02 \mathrm{M} \text { ammonium acetate, } \mathrm{pH} 4.5 \\
\text { (B) ACN }\end{array}$ & \multicolumn{2}{|c|}{$45 \%(B)$} & 5.8 & [99] \\
\hline $\begin{array}{l}\text { Docetaxel pure drug } \\
\text { and injection }\end{array}$ & $\begin{array}{l}\text { UV } \\
(232)\end{array}$ & $\begin{array}{c}\text { Packing L1 } \\
(3.5 \mu \mathrm{m}, 150 \times 4.6 \mathrm{~mm})\end{array}$ & $\begin{array}{l}\text { (A) water } \\
\text { (B) } A C N\end{array}$ & $\begin{array}{c}0 \mathrm{~min} \\
9 \mathrm{~min} \\
39 \mathrm{~min} \\
39.1 \mathrm{~min} \\
49 \mathrm{~min} \\
49.1 \mathrm{~min} \\
60 \mathrm{~min}\end{array}$ & $\begin{array}{l}28 \% \\
28 \% \\
72 \% \\
100 \% \\
100 \% \\
28 \% \\
28 \%\end{array}$ & - & [68] \\
\hline $\begin{array}{l}\text { Docetaxel and } \\
\text { impurities }\end{array}$ & $\begin{array}{l}\text { UV and MS } \\
(230)(807 ; 527) \\
\text { UV } \\
(230)\end{array}$ & $\begin{array}{c}\text { Hypersil BDS } C_{18} \\
(5 \mu \mathrm{m}, 250 \times 4.6 \mathrm{~mm}) \\
\text { YMC Pack ODS-A } \\
(3 \mu \mathrm{m}, 150 \times 4.6 \mathrm{~mm})\end{array}$ & $\begin{array}{l}\text { (A) } 0.02 \mathrm{M} \text { ammonium acetate, } \mathrm{pH} 4.5 \\
\text { (B) ACN } \\
\text { (A) 50:20:30 water:ACN:methanol } \\
\text { (B) } 20: 80 \text { water:ACN }\end{array}$ & $\begin{array}{l}0 \min \\
25 \min \\
26 \min \\
35 \min \end{array}$ & $\begin{array}{c}5 \% \\
60 \% \\
5 \% \\
5 \%\end{array}$ & $\begin{array}{l}26.6 \\
<45\end{array}$ & $\begin{array}{l}{[11]} \\
{[77]}\end{array}$ \\
\hline $\begin{array}{l}\text { Docetaxel and } \\
\text { degradation } \\
\text { products }\end{array}$ & $\begin{array}{l}\text { UV } \\
(230)\end{array}$ & $\begin{array}{c}\text { Hichrom RPB } \\
(5 \mu \mathrm{m}, 250 \times 4.6 \mathrm{~mm})\end{array}$ & $\begin{array}{l}\text { (A) water } \\
\text { (B) ACN }\end{array}$ & $\begin{array}{l}0 \mathrm{~min} \\
15 \mathrm{~min} \\
25 \mathrm{~min} \\
30 \mathrm{~min} \\
35 \mathrm{~min} \\
39 \mathrm{~min} \\
40 \mathrm{~min}\end{array}$ & $\begin{array}{c}35 \% \\
65 \% \\
75 \% \\
95 \% \\
100 \% \\
100 \% \\
35 \%\end{array}$ & $<15$ & {$[3]$} \\
\hline $\begin{array}{l}\text { Docetaxel nanocrystals } \\
\text { surface modified } \\
\text { with transferrin }\end{array}$ & $\begin{array}{l}\text { UV } \\
(230)\end{array}$ & $\begin{array}{c}\text { Intersil OSD-3 } C_{18} \\
(5 \mu \mathrm{m}, 150 \times 4.6 \mathrm{~mm})\end{array}$ & $\begin{array}{l}\text { (A) water } \\
\text { (B) ACN }\end{array}$ & $70 \%$ & & - & [100] \\
\hline \multirow[t]{3}{*}{ Polymeric micelles } & $\begin{array}{l}\text { UV } \\
(232)\end{array}$ & $\begin{array}{l}\text { Waters Nova-Pack } C_{18} \\
(4 \mu \mathrm{m}, 300 \times 3.9 \mathrm{~mm})\end{array}$ & $\begin{array}{l}\text { (A) water } \\
\text { (B) ACN } \\
\text { (C) methanol }\end{array}$ & \multicolumn{2}{|c|}{$\begin{array}{l}\text { Isocratic } \\
48 \%(B)\end{array}$} & - & [101] \\
\hline & $\begin{array}{l}\text { UV } \\
(230)\end{array}$ & $\begin{array}{c}\text { Symmetry } C_{18} \\
(3.5 \mu \mathrm{m}, 150 \times 4.6 \mathrm{~mm})\end{array}$ & $\begin{array}{l}\text { (A) water } \\
\text { (B) ACN }\end{array}$ & \multicolumn{2}{|c|}{$55 \%(B)$} & $<5$ & {$[23]$} \\
\hline & $\begin{array}{l}\text { UV } \\
(230)\end{array}$ & $\begin{array}{l}\text { Dikma Dimansil } C_{18} \\
(5 \mu \mathrm{m}, 150 \times 4.6 \mathrm{~mm})\end{array}$ & $\begin{array}{l}\text { (A) water } \\
\text { (B) ACN }\end{array}$ & \multicolumn{2}{|c|}{$47 \%(B)$} & - & {$[24]$} \\
\hline $\begin{array}{l}\text { Docetaxel-loaded } \\
\text { micelles }\end{array}$ & $\begin{array}{l}\text { UV } \\
(232)\end{array}$ & $\begin{array}{c}\text { Kromasil } C_{18} \\
(3.5 \mu \mathrm{m}, 150 \times 4.6 \mathrm{~mm})\end{array}$ & $\begin{array}{l}\text { (A) ammonium acetate } \\
\text { (B) ACN }\end{array}$ & \multicolumn{2}{|c|}{$45 \%(B)$} & - & [25] \\
\hline $\begin{array}{l}\text { Docetaxel-loaded mixed } \\
\text { micelles }\end{array}$ & $\begin{array}{l}\text { UV } \\
(230) \\
\text { UV } \\
(230)\end{array}$ & $\begin{array}{c}\text { Elite ODS } \mathrm{C}_{18} \\
(5 \mu \mathrm{m}, 250 \times 4.6 \mathrm{~mm}) \\
\text { Capcell pak } \mathrm{C}_{18} \mathrm{MG} \\
(5 \mu \mathrm{m}, 250 \times 4.6 \mathrm{~mm})\end{array}$ & $\begin{array}{l}\text { (A) water } \\
\text { (B) ACN } \\
\text { (A) water } \\
\text { (B) ACN }\end{array}$ & \multicolumn{2}{|c|}{$45(B)$} & $\begin{array}{l}- \\
-\end{array}$ & $\begin{array}{l}{[102]} \\
{[103]}\end{array}$ \\
\hline $\begin{array}{l}\text { Docetaxel-loaded } \\
\text { Polysorbate 80/ } \\
\text { Phospholipid } \\
\text { mixed micelles }\end{array}$ & $\begin{array}{l}\text { UV } \\
(230)\end{array}$ & $\begin{array}{c}\text { Diamonsil } C_{18} \\
(5 \mu \mathrm{m}, 250 \times 4.6 \mathrm{~mm})\end{array}$ & $\begin{array}{l}\text { (A) water } \\
\text { (B) ACN }\end{array}$ & \multicolumn{2}{|c|}{$55 \%(B)$} & $<13$ & [104] \\
\hline $\begin{array}{l}\text { Docetaxel-loaded solid } \\
\text { lipid nanoparticles }\end{array}$ & $\begin{array}{l}\text { UV } \\
(230) \\
\text { UV } \\
(230)\end{array}$ & $\begin{array}{c}\text { Symmetry } C_{18} \\
(5 \mu \mathrm{m}, 250 \times 4.5 \mathrm{~mm}) \\
\text { Diamond } C_{18} \\
(5 \mu \mathrm{m}, 150 \times 4.6 \mathrm{~mm})\end{array}$ & $\begin{array}{l}\text { (A) } 0.2 \% \text { triethylamine, } \mathrm{pH} 6.4 \\
\text { (B) ACN } \\
\text { (A) water } \\
\text { (B) ACN }\end{array}$ & \multicolumn{2}{|c|}{$\begin{array}{l}\text { Isocratic } \\
48 \%(B)\end{array}$} & $\begin{array}{r}11.4 \\
-\end{array}$ & $\begin{array}{l}{[74]} \\
{[105]}\end{array}$ \\
\hline $\begin{array}{l}\text { Docetaxel-loaded lipid } \\
\text { nanoparticles }\end{array}$ & $\begin{array}{l}\text { UV } \\
(230)\end{array}$ & $\begin{array}{c}\text { Inertsil ODS-3 } \\
(4 \mu \mathrm{m}, 150 \times 4.6 \mathrm{~mm})\end{array}$ & $\begin{array}{l}\text { (A) 2-propanol } \\
\text { (B) ACN } \\
\text { (C) water }\end{array}$ & \multicolumn{2}{|c|}{$55 \%(B)$} & 25 & [106] \\
\hline $\begin{array}{l}\text { Docetaxel-loaded } \\
\text { liposomes }\end{array}$ & $\begin{array}{l}\text { UV } \\
(230)\end{array}$ & $\begin{array}{c}\text { Eclipse XDB- } C_{18} \\
(5 \mu \mathrm{m}, 250 \times 4.6 \mathrm{~mm})\end{array}$ & $\begin{array}{l}\text { (A) water } \\
\text { (B) ACN }\end{array}$ & \multicolumn{2}{|c|}{$50 \%(B)$} & - & [107] \\
\hline $\begin{array}{l}\text { Docetaxel-loaded folate- } \\
\text { conjugated PEG- } \\
\text { liposomes }\end{array}$ & $\begin{array}{l}\text { UV } \\
(230)\end{array}$ & $\begin{array}{c}\text { Hypersil } C_{18} \\
(5 \mu \mathrm{m}, 150 \times 4.6 \mathrm{~mm})\end{array}$ & $\begin{array}{l}\text { (A) water } \\
\text { (B) ACN }\end{array}$ & \multicolumn{2}{|c|}{$48 \%(B)$} & - & {$[108]$} \\
\hline $\begin{array}{l}\text { Dual docetaxel/ } \\
\text { superparamagnetic } \\
\text { iron oxide loaded } \\
\text { nanoparticles }\end{array}$ & $\begin{array}{l}\text { UV } \\
(229)\end{array}$ & $\begin{array}{c}\text { Inertsil ODS-3 } \\
(5 \mu \mathrm{m}, 150 \times 4.6 \mathrm{~mm})\end{array}$ & $\begin{array}{l}\text { (A) water } \\
\text { (B) ACN }\end{array}$ & \multicolumn{2}{|c|}{$50 \%(B)$} & - & [109] \\
\hline $\begin{array}{l}\text { Docetaxel-loaded folic } \\
\text { acid conjugated } \\
\text { nanoparticles }\end{array}$ & $\begin{array}{l}\text { UV } \\
(230)\end{array}$ & $\begin{array}{c}\text { Inertsil ODS-3 } \\
(5 \mu \mathrm{m}, 150 \times 4.6 \mathrm{~mm})\end{array}$ & $\begin{array}{l}\text { (A) water } \\
\text { (B) ACN }\end{array}$ & \multicolumn{2}{|c|}{$50 \%(B)$} & - & [110] \\
\hline $\begin{array}{l}\text { Docetaxel-loaded TPGS- } \\
\text { cisplatin prodrug } \\
\text { nanoparticles }\end{array}$ & $\begin{array}{l}\text { UV } \\
(230)\end{array}$ & $\begin{array}{c}\text { Eclipse } \text { XDB- }_{18} \\
(5 \mu \mathrm{m}, 150 \times 4.6 \mathrm{~mm})\end{array}$ & $\begin{array}{l}\text { (A) water } \\
\text { (B) ACN }\end{array}$ & \multicolumn{2}{|c|}{$50 \%(B)$} & - & [111] \\
\hline
\end{tabular}


Table 3. UPLC analysis performed with biological samples.

\begin{tabular}{|c|c|c|c|c|c|c|c|}
\hline \multirow[b]{2}{*}{ Matrix } & \multirow[b]{2}{*}{ Detector } & \multirow[b]{2}{*}{ Column } & \multirow[b]{2}{*}{ Mobile Phase } & \multicolumn{2}{|c|}{ Gradient } & \multirow[b]{2}{*}{$t_{R}(\min )}$. & \multirow[b]{2}{*}{ Ref. } \\
\hline & & & & Time & B & & \\
\hline \multirow[t]{2}{*}{ Human plasma } & $\begin{array}{c}\text { MS/MS } \\
(808.3 ; 527.1)\end{array}$ & $\begin{array}{c}\text { Acquity BEH } \mathrm{C}_{18} \\
(1.7 \mu \mathrm{m}, 50 \times 2.1 \mathrm{~mm})\end{array}$ & $\begin{array}{l}\text { (A) } 0.5 \% \text { formic acid/water, } \mathrm{pH} 2.23 \\
\text { (B) } 0.5 \% \text { formic acid/ACN, pH } 2.24\end{array}$ & $\begin{array}{c}0 \mathrm{~min} \\
0.9 \mathrm{~min} \\
1.6 \mathrm{~min} \\
1.7 \mathrm{~min}\end{array}$ & $\begin{array}{c}10 \% \\
100 \% \\
10 \% \\
10 \%\end{array}$ & 1.35 & {$[16]$} \\
\hline & $\begin{array}{c}\text { MS/MS } \\
(808.3 ; 527.1)\end{array}$ & $\begin{array}{c}\text { Acquity } \mathrm{BEH} \mathrm{C}_{18} \\
(1.7 \mu \mathrm{m}, 50 \times 2.1 \mathrm{~mm})\end{array}$ & $\begin{array}{l}\text { (A) water } / 0.2 \% \text { formic acid } \\
\text { (B) ACN } / 0.2 \% \text { formic acid }\end{array}$ & $\begin{array}{c}0 \min \\
0.9 \min \\
1.6 \mathrm{~min}\end{array}$ & $\begin{array}{c}10 \% \\
100 \% \\
10 \%\end{array}$ & 1.32 & [17] \\
\hline \multirow[t]{3}{*}{ Rat plasma } & $\begin{array}{c}\text { MS/MS } \\
(808.48 ; 527.3)\end{array}$ & $\begin{array}{c}\text { Acquity BEH } \mathrm{C}_{18} \\
(1.7 \mu \mathrm{m}, 50 \times 2.1 \mathrm{~mm})\end{array}$ & $\begin{array}{l}0.1 \% \text { formic acid } \\
\text { (A) ACN } \\
\text { (B) water }\end{array}$ & $\begin{array}{c}0 \mathrm{~min} \\
1.6 \mathrm{~min} \\
2.5 \mathrm{~min} \\
3.0 \mathrm{~min}\end{array}$ & $\begin{array}{l}50 \% \\
35 \% \\
50 \% \\
50 \%\end{array}$ & 1.89 & [112] \\
\hline & $\begin{array}{c}\text { MS/MS } \\
(830.8 ; 549.6)\end{array}$ & $\begin{array}{c}\text { Waters } C_{18} \\
(1.8 \mu \mathrm{m}, 50 \times 2.1 \mathrm{~mm})\end{array}$ & $\begin{array}{l}0.1 \% \text { formic acid } \\
\text { (A) water } \\
\text { (B) methanol }\end{array}$ & $\begin{array}{l}0 \mathrm{~min} \\
1.5 \mathrm{~min} \\
2.5 \mathrm{~min}\end{array}$ & $\begin{array}{l}30 \% \\
80 \% \\
30 \%\end{array}$ & $\sim 1.65$ & [113] \\
\hline & $\begin{array}{c}\text { MS/MS } \\
(830.5 ; 549.3)\end{array}$ & $\begin{array}{c}\text { Venusil } \mathrm{MP} \mathrm{C}_{18} \\
(3.0 \mu \mathrm{m}, 100 \times 2.1 \mathrm{~mm})\end{array}$ & $\begin{array}{l}\text { (A) water } \\
\text { (B) methanol }\end{array}$ & $\begin{array}{c}0 \mathrm{~min} \\
2.5 \mathrm{~min} \\
2.51 \mathrm{~min}\end{array}$ & $\begin{array}{l}75 \% \\
80 \% \\
75 \%\end{array}$ & $<2.5$ & [114] \\
\hline Dog plasma & $\begin{array}{c}\text { MS/MS } \\
(808.25 ; 527.17)\end{array}$ & $\begin{array}{c}\text { Acquity BEH } C_{18} \\
(1.7 \mu \mathrm{m}, 50 \times 2.1 \mathrm{~mm})\end{array}$ & $\begin{array}{l}\text { (A) } \mathrm{ACN} \\
\text { (B) } 5 \mathrm{mM} \text { ammonium acetate/water }\end{array}$ & $\begin{array}{l}0 \mathrm{~min} \\
0.9 \mathrm{~min} \\
2.3 \mathrm{~min}\end{array}$ & $\begin{array}{l}50 \% \\
10 \% \\
50 \%\end{array}$ & $\sim 1.4$ & [115] \\
\hline Rabbit plasma & $\begin{array}{c}\text { MS/MS } \\
(808.4 ; 226.2)\end{array}$ & $\begin{array}{c}\text { Acquity BEH } \mathrm{C}_{18} \\
(1.7 \mu \mathrm{m}, 50 \times 2.1 \mathrm{~mm})\end{array}$ & $\begin{array}{l}\text { (A) } 0.1 \% \text { formic acid } \\
\text { (B) } 49.95: 49.95: 0.1 \text { methanol:ACN:formic acid }\end{array}$ & $\begin{array}{c}0 \mathrm{~min} \\
0.3 \mathrm{~min} \\
1.5 \mathrm{~min} \\
1.7 \mathrm{~min} \\
1.71 \mathrm{~min}\end{array}$ & $\begin{array}{l}10 \% \\
10 \% \\
95 \% \\
10 \% \\
10 \%\end{array}$ & 1.54 & [18] \\
\hline
\end{tabular}

Some methods have been developed for the simultaneous determination of DTX in presence of other drugs by HPLC and UPLC. For instance, Kim et al. ${ }^{[70]}$ developed a RP-HPLC method for the simultaneous determination of DTX and curcumin in rat plasma, using as mobile phase a mixture of ACNwater $(40: 60, \mathrm{v} / \mathrm{v})$ in isocratic elution mode with UV detection at $230 \mathrm{~nm}$, simultaneously for curcumin and DTX, resulting in retention times of 10.47 and $14.26 \mathrm{~min}$, respectively. The method was validated and proved to be selective, sensitive, linear, precise, accurate, robust, and reproducible.

In this same line, da Silva et al. ${ }^{[71]}$ developed an UPLC-MS/ MS method for the simultaneous determination of DTX, cyclophosphamide, doxorubicin, and 5-fluorouracil in order to evaluate the contact of workers exposed to these antineoplastic drugs. Chromatographic separation was done using a SHIMPACK XR-ODC-C ${ }_{18}$ column $(100 \mathrm{~mm} \times 3.0 \mathrm{~mm}$ i.d. $2.2 \mu \mathrm{m}$ particle size; Shimadzu, Kyoto, Japan), using a mobile phase composed of a mixture of $0.1 \% \mathrm{FA}$ and ACN. MS/MS was performed on a triple quadrupole instrument in the MRM mode with transitions at $m / z \quad 129.1>42.1$ for 5-fluorouracil, $544.1>396.9$ for doxorubicin, 261.0 $>140.0$ for cyclophosphamide, and $830.3>304.0$ for DTX.

It has also become common the development of methods for analysis in diverse biological samples, because such complex matrices can interfere with the analyte, requiring a more efficient separation and more sensitive detectors. Therefore, Mortier et al. [72] reported an HPLC-MS/MS method for quantification of DTX and PTX in human plasma and oral fluid (saliva). They used a Merck Purospher Star RP-18 column $(55 \mathrm{~mm} \times 2.0 \mathrm{~mm}$ i.d. $3.0 \mu \mathrm{m}$ particle size; VWR, Leuven, Belgium) and a mobile phase composed of a mixture of (A) $2 \mathrm{mM}$ acetic acid/0.2 mM ammonium acetate in water and (B) $2 \mathrm{mM}$ acetic acid/0.2 mM ammonium acetate in methanol. The MRM transition used was $808.4>526.9 \mathrm{~m} / \mathrm{z}$ and the retention time was $3.5 \mathrm{~min}$. In conclusion, they obtained a sensitive and robust method to quantify taxanes.

Ferrando-Climent et al. ${ }^{[73]}$ on the other hand, innovated and developed a solid-phase extraction (SPE)-UPLC-MS/ MS method for the determination of 10 anticancer drugs, among them DTX, in hospital effluents and wastewater treatment plants (WWTPs), using a method for the screening of human metabolites assisted by information-dependent acquisition (IDA) tool. Chromatographic separation was carried out using an Acquity HSS T3 column $(50 \mathrm{~mm}$ $\times 2.1 \mathrm{~mm}$ i.d. $1.7 \mu \mathrm{m}$ particle size; Waters Corp. Milford, USA). The MS/MS parameters were optimized by MRM positive ionization with transitions at $m / z \quad 808>509$. DTX was found in one hospital effluent and one urban WWTP influent, at concentrations of $97.7 \pm 21.0 \mathrm{ng} \mathrm{L}^{-1}$ and 175.1 $\pm 21.0 \mathrm{ng} \mathrm{\textrm {L } ^ { - 1 }}$, respectively.

Most analytical studies with HPLC employed $\mathrm{C}_{18}$ or $\mathrm{C}_{8}$ reverse phase analytical columns and a water-ACN mixture as the mobile phase, with $\mathrm{pH}$ variations between 3.0 and $6.4,{ }^{[74,75]}$ associated or not with some organic additives such as buffers, acids or bases, to improve selectivity and separation. The gradient elution system was more frequently used than the isocratic elution and the retention times ranged from 1.25 to over $40 \mathrm{~min} .{ }^{[76,77]}$ Altogether, these factors contributed to greater selectivity, better resolution and decreased elution time. However, most studies using delivery systems do not provide all the analytical information, for instance, retention times. And, finally, most analytical studies with UPLC employed $\mathrm{C}_{18}$ columns, commonly with gradient mobile phase composed of $\mathrm{ACN}$, water or methanol, leading to DTX retention times lower than those obtained by HPLC. 


\section{Conclusion}

DTX is an anticancer drug widely used for the treatment of a variety of tumors. As discussed, some physicochemical characteristics, particularly poor water solubility, compromise its pharmacokinetics. As an alternative, herein it was shown a variety of nanocarriers for DTX delivery, which in general, led to better in vitro and in vivo anticancer activity. In this review, we presented several analytical methods for DTX quantification in a variety of matrices, including delivery systems, and biological samples, representing an important step both during formulation development and quality control. Among these methods, immunoassays showed good sensitivity and selectivity, however, the need to use expensive kits limits its use. CE has the advantage of using reduced quantities of samples and solvents, besides having great sensitivity and selectivity, with reduced time of analysis, mostly with the MEEKC method. Despite various methods have been described in this review, the most commonly used method is the reverse phase liquid chromatography, with UV or MS detectors, employing a variety of columns and mobile phases. Finally, the UPLC has gained recent highlight for DTX quantification due to the reduction of mobile phase use and faster analysis.

\section{ORCID}

Gilmar Hanck da Silva (iD) http://orcid.org/0000-0001-8114-6981

Mariza Aires Fernandes (iD http://orcid.org/0000-0003-0412-6439

Lucas Noboru Fatori Trevizan (iD http://orcid.org/0000-0002-9008-1961

Felipe Tita de Lima (iD http://orcid.org/0000-0003-0975-7280

Marlus Chorilli iD http://orcid.org/0000-0002-6698-0545

\section{References}

[1] Aapro, M.; Bruno, R. Early Clinical Studies with Docetaxel. Eur. J. Cancer. 1995, 31, 10-13. DOI: 10.1016/0959-8049(95)00360-U.

[2] Herbst, R. S.; Khuri, F. R. Mode of Action of Docetaxel - A Basis for Combination with Novel Anticancer Agents. Cancer Treat. Rev. 2003, 29, 407-415. DOI: 10.1016/S0305-7372(03)00097-5.

[3] Rao, B. M.; Chakraborty, A.; Srinivasu, M. K.; Devi, M. L.; Kumar, P. R.; Chandrasekhar, K. B.; Srinivasan, A. K.; Prasad, A. S.; Ramanatham, J. A Stability-Indicating HPLC Assay Method for Docetaxel. J. Pharm. Biomed. Anal. 2006, 41, 676-681. DOI:10.1016/j. jpba.2006.01.011.

[4] U. S. Food and Drug Administration/Center for Drug Evaluation and Research. New Drug Application - Review (FDA-NDA 20449/S-035). Taxotere ${ }^{\circledR}$-Docetaxel. 2006. https://www.accessdata. fda.gov/drugsatfda_docs/nda/2006/020449Orig1s035.pdf (accessed Dec 17, 2017).

[5] Valicherla, G. R.; Dave, K. M.; Syed, A. A.; Riyazuddin, M.; Gupta, A. P.; Singh, A.; Wahajuddin; Mitra, K.; Datta, D.; Gayen, J. R. Formulation Optimization of Docetaxel Loaded Self-Emulsifying Drug Delivery System to Enhance Bioavailability and Anti-Tumor Activity. Sci. Rep. 2016, 6, 26895. DOI: 10.1038/srep26895.

[6] Yvon, A. -M. C.; Wadsworth, P.; Jordan, M. A. Taxol Suppresses Dynamics of Individual Microtubules in Living Human Tumor Cells. Mol. Biol. Cell. 1999, 10, 947-959. DOI: 10.1091/mbc.10.4.947.

[7] Arbuck, S. G.; Blaylock, B. A. Taxol: Clinical Results and Current Issues in Development. In Taxol: Science and Applications, Suffness, M., Eds.; CRC Press: Boca Raton, 1995; pp 379-416.

[8] Ojima, I.; Slater, J. C.; Kuduk, S. D.; Takeuchi, C. S.; Gimi, R. H.; Sun, C. M.; Park, Y. H.; Pera, P.; Veith, J. M.; Bernacki, R. J. Syntheses and Structure-Activity Relationships of Taxoids Derived from 14 $\beta$-Hydroxy-10-Deacetylbaccatin III. J. Med. Chem. 1997, 40, 267-278. DOI: 10.1021/jm960563e.
[9] Tan, Q.; Liu, X.; Fu, X.; Li, Q.; Dou, J.; Zhai, G. Current Development in Nanoformulations of Docetaxel. Expert Opin. Drug Deliv. 2012, 9, 975-990. DOI: 10.1517/17425247.2012.696606.

[10] Rafiei, P.; Haddadi, A. Docetaxel-Loaded PLGA and PLGA-PEG Nanoparticles for Intravenous Application: Pharmacokinetics and Biodistribution Profile. Int. J. Nanomedicine. 2017, 12, 935-947. DOI: $10.2147 /$ IJN.S121881.

[11] Vasu Dev, R.; Moses Babu, J.; Vyas, K.; Sai Ram, P.; Ramachandra, P.; Sekhar, N. M.; Mohan Reddy, D. N.; Srinivasa Rao, N. Isolation and Characterization of Impurities in Docetaxel. J. Pharm. Biomed. Anal. 2006, 40, 614-622. DOI: 10.1016/j.jpba.2005.10.037.

[12] Yin, Y. M.; De Cui, F.; Mu, C. F.; Choi, M. K.; Kim, J. S.; Chung, S. J.; Shim, C. K.; Kim, D. D. Docetaxel Microemulsion for Enhanced Oral Bioavailability: Preparation and in Vitro and in Vivo Evaluation. J. Control. Release. 2009, 140, 86-94. DOI: 10.1016/j. jconrel.2009.08.015.

[13] Cline, D. J.; Zhang, H.; Lundell, G. D.; Harney, R. L.; Riaz, H. K.; Jarrah, J.; Li, Y.; Miyazaki, M.; Courtney, J. B.; Baburina, I.; Salamone, S. J. An Automated Nanoparticle-Based Homogeneous Immunoassay for Determining Docetaxel Concentrations in Plasma. Ther. Drug Monit. 2013, 35, 803-808. DOI: 10.1097/ FTD.0b013e31829617ea.

[14] Geng, C.; Li, P.; Chen, X.; Yuan, G.; Guo, N.; Liu, H.; Zhang, R. Comparison of the Docetaxel Concentration in Human Plasma Measured with Liquid Chromatography-Tandem Mass Spectrometry (Lc-Ms/Ms) and a Nanoparticle Immunoassay and Clinical Applications of that Assay. Biosci. Trends. 2017, 11, 202-208. DOI 10.5582/bst.2017.01029.

[15] Shakalisava, Y.; Regan, F. CE Separation Approaches for Combinations of Anthracyclines and Taxanes. Electrophoresis. 2009, 30, 3110-3113. DOI: 10.1002/elps.200900097.

[16] Du, P.; Li, N.; Wang, H.; Yang, S.; Song, Y.; Han, X.; Shi, Y. Development and Validation of a Rapid and Sensitive UPLC-MS/MS Method for Determination of Total Docetaxel from a Lipid Microsphere Formulation in Human Plasma. J. Chromatogr. B. 2013, 926, 101-107. DOI: 10.1016/j.jchromb.2013.02.006.

[17] Du, P.; Han, X.; Li, N.; Wang, H.; Yang, S.; Song, Y.; Shi, Y. Development and Validation of an Ultrafiltration-UPLC-MS/MS Method for Rapid Quantification of Unbound Docetaxel in Human Plasma. J. Chromatogr. B. 2014, 967, 28-35. DOI: $10.1016 /$ j. jchromb.2014.07.007.

[18] Wang, J.; Lan, Z.; Zhang, L.; Guo, H.; Liu, Z.; Yu, Y. A. Rapid and Sensitive UPLC-MS/MS Method for Determination of Docetaxel in Rabbit Plasma: Pharmacokinetic Study ofcNew Lung-Targeting Docetaxel Liposome at Low Dose. Cell Biochem. Biophys. 2015, 73, 623-629. DOI: 10.1007/s12013-015-0639-z.

[19] Garg, M. B.; Ackland, S.P. Simple and Sensitive High-Performance Liquid Chromatography Method for the Determination of Docetaxel in Human Plasma or Urine. J. Chromatogr. B. Biomed. Sci. Appl. 2000, 748, 383-388. DOI: 10.1016/S0378-4347(00)00356-X.

[20] Ehrlichova, M.; Vaclavikova, R.; Ojima, I.; Pepe, A.; Kuznetsova, L. V.; Chen, J.; Truksa, J.; Kovar, J.; Gut, I. Transport and Cytotoxicity of Paclitaxel, Docetaxel, and Novel Taxanes in Human Breast Cancer Cells. Naunyn. Schmiedebergs. Arch. Pharmacol. 2005, 372, 95 105. DOI: 10.1007/s00210-005-1080-4.

[21] Andersen, A.; Warren, D. J.; Brunsvig, P. F.; Aamdal, S.; Kristensen, G. B.; Olsen, H. High Sensitivity Assays for Docetaxel and Paclitaxel in Plasma using Solid-Phase Extraction and High-Performance Liquid Chromatography with UV Detection. BMC Clin. Pharmacol. 2006, 6 (2), 1-10. DOI: 10.1186/1472-6904-6-2.

[22] Navarrete, A.; Martínez-Alcázar, M. P.; Durán, I.; Calvo, E.; Valenzuela, B.; Barbas, C.; García, A. Simultaneous Online SPE-HPLCMS/MS Analysis of Docetaxel, Temsirolimus and Sirolimus in Whole Blood and Human Plasma. J. Chromatogr. B. 2013, 921-922, 35-42. DOI: 10.1016/j.jchromb.2013.01.017.

[23] Shi, J.; Zhang, J.; Shen, Y.; Tang, L.; Zhao, J.; Tu, J.; Tian, Y.; Feng, Y. Arginine-Stabilized mPEG-PDLLA (50/50) Polymeric Micelles of Docetaxel by Electrostatic Mechanism for Tumor-Targeted Delivery. Drug Deliv. 2013, 7544, 1-14. DOI: 10.3109/ 10717544.2013.849779. 
[24] Yang, M.; Ding, Y.; Zhang, L.; Qian, X.; Jiang, X.; Liu, B. Novel Thermosensitive Polymeric Micelles for Docetaxel Delivery. $J$. Biomed. Mater. Res. Part A. 2007, 81A, 847-857. DOI: 10.1002/ jbm.a.31129.

[25] Zhang, L.; Tan, L.; Chen, L.; Chen, X.; Long, C.; Peng, J.; Qian, Z. A Simple Method to Improve the Stability of Docetaxel Micelles. Sci. Rep. 2016, 6, 2-11. DOI: 10.1038/srep36957.

[26] Clarke, S. J.; Rivory, L. P. Clinical Pharmacokinetics of Docetaxel. Clin. Pharmacokinet. 1999, 36, 99-114. DOI: 10.2165/00003088199936020-00002.

[27] Vella-Zarb, L.; Dinnebier, R. E.; Baisch, U. The Devil is in the Detail: A rare H-Bonding Motif in new forms of Docetaxel. Cryst. Growth Des. 2013, 13, 4402-4410. DOI: 10.1021/cg400814a.

[28] De Weger, V. A.; Beijnenb, J. H.; Schellensa, J. H. M. Cellular and Clinical Pharmacology of the Taxanes Docetaxel and Paclitaxel - A Review. Anticancer. Drugs. 2014, 25, 488-494. DOI: 10.1097/ CAD.0000000000000093.

[29] Kenmotsu, H.; Tanigawara, Y. Pharmacokinetics, Dynamics and Toxicity of Docetaxel: Why the Japanese Dose Differs from the Western Dose. Cancer Sci. 2015, 106, 497-504. DOI: 10.1111/ cas.12647.

[30] Baker, S. D.; Li, J.; Ten Tije, A. J.; Figg, W. D.; Graveland, W.; Verweij, J.; Sparreboom, A. Relationship of Systemic Exposure to Unbound Docetaxel and Neutropenia. Clin. Pharmacol. Ther. 2005, 77, 43-53. DOI: 10.1016/j.clpt.2004.09.005.

[31] Rosing, H.; Lustig, V.; van Warmerdam, L. J.; Huizing, M. T.; ven Bokkel Huinink, W. W.; Schellens, J. H.; Rodenhuis, S.; Bult, A.; Beijnen, J. H. Pharmacokinetics and Metabolism of Docetaxel Administered as a 1-h Intravenous Infusion. Cancer Chemother. Pharmacol. 2000, 45, 213-8. DOI: 10.1007/s002800050032.

[32] Baker, S. D.; Verweij, J.; Cusatis, G. A.; Van Schaik, R. H.; Marsh, S.; Orwick, S. J.; Franke, R. M.; Hu, S.; Schuetz, E. G.; Lamba, V.; Messersmith, W. A.; Wolff, A. C.; Carducci, M. A.; Sparreboom, A. Pharmacogenetic Pathway Analysis of Docetaxel Elimination. Clin. Pharmacol. Ther. 2009, 85, 155-163. DOI: 10.1038/clpt.2008.95.

[33] Baker, S. D.; Sparreboom, A.; Verweij, J. Clinical Pharmacokinetics of Docetaxel: Recent Developments. Clin. Pharmacokinet. 2006, 45, 235-252. DOI: 10.2165/00003088-200645030-00002.

[34] van Oosterom, A. T.; Schriivers, D. Docetaxel (Taxotere ${ }^{\circledR}$ ), a Review of Preclinical and Clinical Experience. Part II. Anticancer Drugs. 1995, 6, 356-368. DOI: 10.1097/00001813-199506000-00002.

[35] Royer, I.; Monsarrat, B.; Sonnier, M.; Wright, M.; Cresteil, T. Metabolism of Docetaxel by Human Cytochromes P450: Interactions with Paclitaxel and Other Antineoplastic Drugs. Cancer Res. 1996, 56, 58-65.

[36] Hooker, A. C.; Ten Tije, A. J.; Carducci, M. A.; Weber, J.; GarrettMayer, E.; Gelderblom, H.; McGuire, W. P.; Verweij, J.; Karlsson, M. O.; Baker, S. D. Population Pharmacokinetic Model for Docetaxel in Patients with Varying Degrees of Liver Function: Incorporating Cytochrome $\mathrm{P} 450$ 3A Activity Measurements. Clin. Pharmacol. Ther. 2008, 84, 111-118. DOI: 10.1038/sj.clpt.6100476.

[37] Morris, P. G.; Fornier, M. N. Microtubule Active Agents: Beyond the Taxane Frontier. Clin. Cancer Res. 2008, 14, 7167-7172. DOI: 10.1158/1078-0432.CCR-08-0169.

[38] Jordan, M. A.; Wilson, L. Microtubules as a Target for Anticancer Drugs. Nat. Rev. Cancer. 2004, 4, 253-265. DOI: 10.1038/nr1317.

[39] Mollinedo, F.; Gajate, C. Microtubules, Microtubule-Interfering Agents and Apoptosis. Apoptosis. 2003, 8, 413-450. DOI: 10.1023/ A:1025513106330.

[40] Altmann, K. -H.; Gertsch, J. Anticancer Drugs from Nature-Natural Products as a Unique Source of New Microtubule-Stabilizing Agents. Nat. Prod. Rep. 2007, 24, 327-357. DOI: 10.1039/B515619J.

[41] Dumontet, C.; Sikic, B. I. Mechanisms of Action of and Resistance to Antitubulin Agents: Microtubule Dynamics, Drug Transport, and Cell Death. J Clin Oncol. 1999, 17, 1061-1070. DOI: 10.1200/ JCO.1999.17.3.1061.

[42] Sissung, T. M.; Baum, C. E.; Deeken, J.; Price, D. K.; Aragon-Ching, J.; Steinberg, S. M.; Dahut, W.; Sparreboom, A.; Figg, W. D. ABCB1 Genetic Variation Influences the Toxicity and Clinical Outcome Of
Patients With Androgen-Independent Prostate Cancer Treated With Docetaxel. Clin. Cancer Res. 2008, 14, 4543-4549. DOI: 10.1158/1078-0432.CCR-07-4230.

[43] Vacca, F.; Ribatti, A.; Iurlaro, D.; Merchionne, M.; Nico, F.; Ria, B.; Dammacco, R. Docetaxel Versus Paclitaxel for Antiangiogenesis. J. Hematother. Stem Cell Res. 2002, 11, 103-118. DOI: 10.1089/ 152581602753448577

[44] Rowinsky, E. K. The Development and Clinical Utility of the Taxane Class of Antimicrotubule Chemotherapy Agents. Annu. Rev. Med. 1997, 48, 353-74. DOI: 10.1146/annurev.med.48.1.353.

[45] Montero, A.; Fossella, F.; Hortobagyi, G.; Valero, V. Docetaxel for Treatment of Solid Tumours: A Systematic Review of Clinical Data. Lancet. Oncol. 2005, 6, 229-39. DOI: 10.1016/S1470-2045(05) 70094-2.

[46] Benelli, R.; Monteghirfo, S.; Balbi, C.; Barboro, P.; Ferrari, N. Novel Antivascular Efficacy of Metronomic Docetaxel Therapy in Prostate Cancer: hnRNP K as a Player. Int. J. Cancer. 2009, 124, 2989-2996. DOI: $10.1002 / \mathrm{ijc} .24305$.

[47] Engels, F. K.; Mathot, R. A. A.; Verweij, J. Alternative Drug Formulations of Docetaxel: A Review. Anticancer Drugs. 2007, 18, 95-103. DOI: 10.1097/CAD.0b013e3280113338.

[48] Sanna, V.; Sechi, M. Nanoparticle Therapeutics for Prostate Cancer Treatment. Maturitas. 2012, 73, 27-32. DOI: 10.1016/j. maturitas.2012.01.016.

[49] Wicki, A.; Witzigmann, D.; Balasubramanian, V.; Huwyler, J. Nanomedicine in Cancer Therapy: Challenges, Opportunities, and Clinical Applications. J. Control. Release. 2015, 200, 138-157. DOI: 10.1016/j.jconrel.2014.12.030.

[50] Maeda, H.; Wu, J.; Sawa, T.; Matsumura, Y.; Hori, K. Tumor Vascular Permeability and the Epr Effect in Macromolecular Therapeutics: A Review. J. Control. Release. 2000, 65, 271-284. DOI: 10.1016/ S0168-3659(99)00248-5.

[51] Stefanutti, E.; Papacci, F.; Sennato, S.; Bombelli, C.; Viola, I.; Bonincontro, A.; Bordi, F.; Mancini, G.; Gigli, G.; Risuleo, G. Cationic Liposomes Formulated with DMPC and a Gemini Surfactant Traverse the Cell Membrane without Causing a Significant Bio-Damage. Biochim. Biophys. Acta - Biomembr. 2014, 1838, 2646-2655. DOI: 10.1016/j.bbamem.2014.05.026.

[52] Zhang, H.; Gong, W.; Wang, Z. Y.; Yuan, S. J.; Xie, X. Y.; Yang, Y. F.; Yang, Y.; Wang, S. S.; Yang, D. X.; Xuan, Z. X.; Mei, X. G. Preparation, Characterization, and Pharmacodynamics of Thermosensitive Liposomes Containing Docetaxel. J. Pharm. Sci. 2014, 103, 2177-2183. DOI: 10.1002/jps.24019.

[53] Chang, M.; Lu, S.; Zhang, F.; Zuo, T.; Guan, Y.; Wei, T.; Shao, W.; Lin, G. RGD-Modified pH-Sensitive Liposomes for Docetaxel Tumor Targeting. Colloids Surf B Biointerfaces. 2015, 129, 175-182. DOI: 10.1016/j.colsurfb.2015.03.046.

[54] Chu, C.; Xu, P.; Zhao, H.; Chen, Q.; Chen, D.; Hu, H.; Zhao, X.; Qiao, M. Effect of Surface Ligand Density on Cytotoxicity and Pharmacokinetic Profile of Docetaxel Loaded Liposomes. Asian J. Pharm. Sci. 2016, 11, 655-661. DOI: 10.1016/j.ajps.2016.04.001.

[55] Yoon, H. Y.; Kwak, S. S.; Jang, M. H.; Kang, M. H.; Sung, S. W.; Kim, C. H.; Kim, S. R.; Yeom, D. W.; Kang, M. J.; Choi, Y. W. DocetaxelLoaded RIPL Peptide (IPLVVPLRRRRRRRRC)-Conjugated Liposomes: Drug Release, Cytotoxicity, and Antitumor Efficacy. Int. J. Pharm. 2017, 523, 229-237. DOI: 10.1016/j.ijpharm.2017.03.045.

[56] Pereira, S.; Egbu, R.; Jannati, G.; Al-Jamal, W. T. Docetaxel-Loaded Liposomes: The Effect of Lipid Composition and Purification on Drug Encapsulation and in Vitro Toxicity. Int. J. Pharm. 2016, 514, 150-159. DOI: 10.1016/j.ijpharm.2016.06.057.

[57] Pan, Y.; Tikekar, R. V.; Nitin, N. Distribution of a Model Bioactive Within Solid Lipid Nanoparticles and Nanostructured Lipid Carriers Influences its Loading Efficiency and Oxidative Stability. Int. J. Pharm. 2016, 511, 322-330. DOI: 10.1016/j.ijpharm.2016.07.019.

[58] Liu, D.; Liu, Z.; Wang, L.; Zhang, C.; Zhang, N. Nanostructured Lipid Carriers as Novel Carrier for Parenteral Delivery of Docetaxel. Colloids Surf B Biointerfaces. 2011, 85, 262-269. DOI: 10.1016/j. colsurfb.2011.02.038.

[59] Zhang, F.; Chang, M.; Yu, Y.; Zhang, Y.; Liu, G.; Wei, T.; Zuo, T.; Guan, Y.; Lin, G.; Zhao, Z. Preparation and Evaluation of Lipid 
Emulsified Docetaxel-Loaded Nanoparticles. J. Pharm. Pharmacol. 2015, 67, 1546-1555. DOI: 10.1111/jphp.12472.

[60] Raza, K.; Kumar, N.; Misra, C.; Kaushik, L.; Guru, S. K.; Kumar, P.; Malik, R.; Bhushan, S.; Katare, O. P. Dextran-PLGA-Loaded Docetaxel Micelles with Enhanced Cytotoxicity and Better Pharmacokinetic Profile. Int. J. Biol. Macromol. 2016, 88, 206-212. DOI: 10.1016/j.ijbiomac.2016.03.064.

[61] Petrilli, R.; Eloy, J.O.; Marchetti, J.M.; Lopez, R.V.; Lee, R.J. Targeted Lipid Nanoparticles for Antisense Oligonucleotide Delivery. Curr. Pharm. Biotechnol. 2014, 15, 847-855 DOI: 10.2174/ 1389201015666141020155834.

[62] Nateghian, N.; Goodarzi, N.; Amini, M.; Atyabi, F.; Khorramizadeh, M. R.; Dinarvand, R. Biotin/Folate-Decorated Human Serum Albumin Nanoparticles of Docetaxel: Comparison of Chemically Conjugated Nanostructures and Physically Loaded Nanoparticles for Targeting of Breast Cancer. Chem. Biol. Drug Des. 2016, 87, 69-82 DOI: $10.1111 /$ cbdd.12624.

[63] Raju, A.; Muthu, M.S.; Feng, S.S. Trastuzumab-Conjugated Vitamin E TPGS Liposomes for Sustained and Targeted Delivery of Docetaxel. Expert Opin. Drug Deliv. 2013, 10, 747-760 DOI: 10.1517/ 17425247.2013.777425.

[64] Kulhari, H.; Pooja, D.; Shrivastava, S. N. V. G. M; Sistla, R. Peptide Conjugated Polymeric Nanoparticles as a Carrier for Targeted Delivery of Docetaxel. Colloids Surf B Biointerfaces. 2014, 117, 166173. DOI: $10.1016 /$ j.colsurfb.2014.02.026.

[65] Patil, R.; Deshmukh, T.; Patil, V.; Khandelwal, K. Review on Analytical Method Development and Validation. Res. Rev. J. Pharm. Anal. 2014, 3, 1-10.

[66] Siddiqui, M. R.; AlOthman, Z. A.; Rahman, N. Analytical Techniques in Pharmaceutical Analysis: A Review. Arab. J. Chem. 2017, 10, S1409-S1421. DOI: 10.1016/j.arabjc.2013.04.016.

[67] Suntornsuk, L. Capillary Electrophoresis in Pharmaceutical Analysis: A Survey on Recent Applications. J. Chromatogr. Sci. 2007, 45, 559-77. DOI: 10.1023/A:1012018709641.

[68] United States Pharmacopeia Convention. The United States pharmacopeia, The national formulary. USP 35nd rev., NF 30th ed. Vol 2. Rockville (MD): The Convention; 2012.

[69] Maldaner, L.; Jardim, I. C. S. F. O Estado Da Arte Da Cromatografia Líquida De Ultra Eficiência. Quim. Nova. 2009, 32, 214-222. DOI: 10.1590/S0100-40422009000100036.

[70] Kim, D. W.; Yousaf, A. M.; Li, D. X.; Kim, J. O.; Yong, C. S.; Cho, K. H.; Choi, H. G. Development of RP-HPLC Method for Simultaneous Determination of Docetaxel and Curcumin in Rat Plasma: Validation and Stability. Asian J. Pharm. Sci. 2017, 12, 105-113. DOI: 10.1016/j.ajps.2016.08.002.

[71] da Silva, C. B. P.; Julio, I. P.; Donadel, G. E.; Martins, I. UPLC-MS/ MS Method for Simultaneous Determination of Cyclophosphamide, Docetaxel, Doxorubicin and 5-Fluorouracil in Surface Samples. J. Pharmacol. Toxicol. Methods. 2016, 82, 68-73. DOI: 10.1016/j.vascn.2016.08.004.

[72] Mortier, K. A.; Renard, V.; Verstraete, A. G.; Lambert, W. E.; Van Gussem, A.; Van Belle, S. Development and Validation of a Liquid Chromatography- Tandem Mass Spectrometry Assay for the Quantification of Docetaxel and Paclitaxel in Human Plasma and Oral Fluid. Anal. Chem. 2005, 77, 4677-4683. DOI: 10.1021/ac0500941.

[73] Ferrando-Climent, L.; Rodriguez-Mozaz, S.; Barceló, D. Development of a UPLC-MS/MS Method for the Determination of Ten Anticancer Drugs in Hospital and Urban Wastewaters, and its Application for the Screening of Human Metabolites Assisted by Information-Dependent Acquisition Tool (Ida) in Sewage Samples. Anal. Bioanal. Chem. 2013, 405, 5937-5952. DOI: 10.1007/s00216013-6794-4.

[74] Venishetty, V. K.; Parikh, N.; Sistla, R.; Ahmed, F. J.; Diwan, P. V. Application of Validated RP-HPLC Method for Simultaneous Determination of Docetaxel and Ketoconazole in Solid Lipid Nanoparticles. J. Chromatogr. Sci. 2011, 49, 136-141. DOI: 10.1093/ chrsci/49.2.136

[75] Ardiet, C. J.; Tranchand, B.; Zanetta, S.; Guillot, A.; Bernard, E.; Peguy, M.; Rebattu, P.; Droz, J. P. A Sensitive Docetaxel Assay in
Plasma by Solid-Phase Extraction and High Performance Liquid Chromatography - UV Detection: Validation and Suitability in Phase I Clinical Trial Pharmacokinetics. Invest. New Drugs. 1999, 17, 325-333. DOI: 10.1023/A:1006327302041.

[76] Patro, V. J.; Rao, R. N.; Tripathy, N. K. Liquid Chromatography Tandem Mass Spectrometry Method for Determination of Docetaxel in K3EDTA Human Plasma. Int. J. Pharm. Chem. Biol. Sci. 2014, 4, 711-716.

[77] Malleswara Reddy, A.; Banda, N.; Govind Dagdu, S.; Rao, D. V.; Kocherlakota, C. S.; Krishnamurthy, V. Evaluation of the Pharmaceutical Quality of Docetaxel Injection Using New Stability Indicating Chromatographic Methods for Assay and Impurities. Sci. Pharm. 2010, 78, 215-231. DOI: 10.3797/scipharm.0912-14.

[78] Corona, G.; Elia, C.; Casetta, B.; Frustaci, S.; Toffoli, G. HighThroughput Plasma Docetaxel Quantification by Liquid Chromatography-Tandem Mass Spectrometry. Clin. Chim. Acta. 2011, 412, 358-364. DOI: 10.1016/j.cca.2010.11.010.

[79] Ciccolini, J.; Catalin, J.; Blachon, M. F.; Durand, A. Rapid High-Performance Liquid Chromatographic Determination of Docetaxel (Taxotere) in Plasma Using Liquid-Liquid Extraction. J. Chromatogr. B Biomed. Sci. Appl. 2001, 759, 299-306. DOI: 10.1016/S03784347(01)00238-9.

[80] Baker, S. D.; Zhao, M.; He, P.; Carducci, M. A.; Verweij, J.; Sparreboom, A. Simultaneous Analysis of Docetaxel and the Formulation Vehicle Polysorbate 80 in Human Plasma by Liquid Chromatography/Tandem Mass Spectrometry. Anal. Biochem. 2004, 324, 276284. DOI: 10.1016/j.ab.2003.09.038.

[81] Guitton, J.; Cohen, S.; Tranchand, B.; Vignal, B.; Droz, J. P.; Guillaumont, M.; Manchon, M.; Freyer, G. Quantification of Docetaxel and its Main Metabolites in Human Plasma by Liquid Chromatography/Tandem Mass Spectrometry. Rapid Commun. Mass Spectrom. 2005, 19, 2419-2426. DOI: 10.1002/rcm. 2072.

[82] Hendrikx, J. J. M. A.; Hillebrand, M. J. X.; Thijssen, B.; Rosing, H.; Schinkel, A. H.; Schellens, J. H. M.; Beijnen, J. H. A Sensitive Combined Assay for the Quantification of Paclitaxel, Docetaxel and Ritonavir in Human Plasma Using Liquid Chromatography Coupled with Tandem Mass Spectrometry. J. Chromatogr. B. 2011, 879, 2984-2990. DOI: 10.1016/j.jchromb.2011.08.034.

[83] Kuppens, I. E. L. M.; van Maanen, M. J.; Rosing, H.; Schellens, J. H. M.; Beijnen, J. H. Quantitative Analysis of Docetaxel in Human Plasma Using Liquid Chromatography Coupled with Tandem Mass Spectrometry. Biomed. Chromatogr. 2005, 19, 355-361. DOI 10.1002/bmc.457.

[84] Loos, W. J.; Verweij, J.; Nooter, K.; Stoter, G.; Sparreboom, A. Sensitive Determination of Docetaxel in Human Plasma by Liquid-Liquid Extraction and Reversed-Phase High-Performance Liquid Chromatography. J. Chromatogr. B. Biomed. Sci. Appl. 1997, 693, 437-441. DOI: 10.1016/S0378-4347(97)00089-3.

[85] Zufía López, L.; Aldaz Pastor, A.; Aramendia Beitia, J. M.; Arrobas Velilla, J.; Giraldez Deiró, J. Determination of Docetaxel and Paclitaxel in Human Plasma by High-Performance Liquid Chromatography: Validation and Application to Clinical Pharmacokinetic Studies. Ther. Drug Monit. 2006, 28, 199-205. DOI: 10.1097/01. ftd.0000189903.46802.1f.

[86] Parise, R. A.; Ramanathan, R. K.; Zamboni, W. C.; Egorin, M. J. Sensitive Liquid Chromatography-Mass Spectrometry Assay for Quantitation of Docetaxel and Paclitaxel in Human Plasma. J. Chromatogr. B Anal. Technol. Biomed. Life Sci. 2003, 783, 231-236. DOI: 10.1016/S1570-0232(02)00659-1.

[87] Rosing, H.; Lustig, V.; Koopman, F. P.; ten Bokkel Huinink, W. W.; Beijnen, J. H. Bio-Analysis of Docetaxel and Hydroxylated Metabolites in Human Plasma by High-Performance Liquid Chromatography and Automated Solid-Phase Extraction. J. Chromatogr. B. Biomed. Sci. Appl. 1997, 696, 89-98. DOI: 10.1016/S0378-4347(97) 00209-0.

[88] Rouini, M. R.; Lotfolahi, A.; Stewart, D. J.; Molepo, J. M.; Shirazi, F. H.; Vergniol, J. C.; Tomiak, E.; Delorme, F.; Vernillet, L.; Giguere, M.; Goel, R. A Rapid Reversed Phase High Performance Liquid Chromatographic Method for the Determination of Docetaxel (Taxotere ${ }^{\circledR}$ ) in Human Plasma Using a Column Switching 
Technique. J. Pharm. Biomed. Anal. 1998, 17, 1243-1247. DOI: 10.1016/S0731-7085(97)00233-1.

[89] Vergniol, J. C.; Bruno, R.; Montay, G.; Frydman, A. Determination of Taxotere in Human Plasma by a Semi-Automated High-Performance Liquid Chromatographic Method. J. Chromatogr. B Biomed. Sci. Appl. 1992, 582, 273-278. DOI: 10.1016/0378-4347(92)80333-L.

[90] Wang, L. Z.; Goh, B. C.; Grigg, M. E.; Lee, S. C.; Khoo, Y. M.; Lee, H. S. A Rapid and Sensitive Liquid Chromatography/Tandem Mass Spectrometry Method for Determination of Docetaxel in Human Plasma. Rapid Commun. Mass Spectrom. 2003, 17, 1548-1552. DOI: $10.1002 / \mathrm{rcm} .1091$.

[91] Yamaguchi, H.; Fujikawa, A.; Ito, H.; Tanaka, N.; Furugen, A.; Miyamori, K.; Takahashi, N.; Ogura, J.; Kobayashi, M.; Yamada, T.; Mano, N.; Iseki, K. A Rapid and Sensitive LC/ESI-MS/MS Method for Quantitative Analysis of Docetaxel in Human Plasma and its Application to a Pharmacokinetic Study. J. Chromatogr. B. 2012, 893-894, 157-161. DOI: 10.1016/j.jchromb.2012.02.003.

[92] Hou, W.; Watters, J. W.; McLeod, H. L. Simple and Rapid Docetaxel Assay in Plasma by Protein Precipitation and High-Performance Liquid Chromatography-Tandem Mass Spectrometry. J. Chromatogr. B. 2004, 804, 263-267. DOI: 10.1016/j.jchromb.2004.01.021.

[93] Huang, Q.; Wang, G.-J.; Sun, J.-G.; Hu, X.-L.; Lu, Y.-H.; Zhang, Q. Simultaneous Determination of Docetaxel and Ketoconazole in Rat Plasma by Liquid Chromatography/Electrospray Ionization Tandem Mass Spectrometry. Rapid Commun. Mass Spectrom. 2007, 21, 1009-1018. DOI: 10.1002/rcm.2903.

[94] Marchettini, P.; Stuart, O. A.; Mohamed, F.; Yoo, D.; Sugarbaker, P. H. Docetaxel: Pharmacokinetics and Tissue Levels After Intraperitoneal and Intravenous Administration in a Rat Model. Cancer Chemother. Pharmacol. 2002, 49, 499-503. DOI: 10.1007/s00280-0020439-1.

[95] Xu, Q.; Zhang, N.; Yin, X.; Wang, M.; Shen, Y.; Xu, S.; Zhang, L.; $\mathrm{Gu}, \mathrm{Z}$. Development and Validation of a Nylon6 Nanofibers MatBased Spe Coupled with Hplc Method for the Determination of Docetaxel in Rabbit Plasma and its Application to the Relative Bioavailability Study. J. Chromatogr. B. 2010, 878, 2403-2408. DOI: 10.1016/j.jchromb.2010.07.011.

[96] Zhao, L.; Wei, Y.-M; Zhong, X.-D; Liang, Y.; Zhang, X.-M; Li, W.; Li, B.-B.; Wang, Y.; Yu, Y. PK and Tissue Distribution of Docetaxel in Rabbits After i.v. Administration of Liposomal and Injectable Formulations. J. Pharm. Biomed. Anal. 2009, 49, 989-996. DOI: 10.1016/j.jpba.2009.01.016

[97] Devi, G. K. Method Development and Validation of RP-HPLC Method for Docetaxel API Along with its Stability Studies. Int. J. Med. Nanotec. 2015, 2 (5), 282-288.

[98] Kumar, D.; Tomar, R. S.; Deolia, S. K.; Mitra, M.; Mukherjee, R.; Burman, A. C. Isolation and Characterization of Degradation Impurities in Docetaxel Drug Substance and its Formulation. J. Pharm. Biomed. Anal. 2007, 43, 1228-1235. DOI: 10.1016/j. jpba.2006.10.015.

[99] Kumar, K. N.; Raju, N. A.; Raju, S. S.; Ramarao, B.; Mukkanti, K. Estimation of Docetaxel in Parenterals by RP-HPLC. Asian J. Chem. 2010, 22, 7513-7518.

[100] Choi, J. S.; Park, J. S. Development of docetaxel Nanocrystals Surface Modified with Transferrin for Tumor Targeting. Drug Des. Devel. Ther. 2017, 11, 17-26. DOI: 10.2147/DDDT.S122984.

[101] Elsabahy, M.; Perron, M. É.; Bertrand, N.; Yu, G. E.; Leroux, J. C. Solubilization of Docetaxel in Poly(Ethylene Oxide)-Block-Poly (Butylene/Styrene Oxide) Micelles. Biomacromolecules. 2007, 8, 2250-2257. DOI: $10.1021 / \mathrm{bm} 070226 \mathrm{v}$.

[102] Dou, J.; Zhang, H.; Liu, X.; Zhang, M.; Zhai, G. Preparation and Evaluation in Vitro and in Vivo of Docetaxel Loaded Mixed
Micelles for Oral Administration. Colloids Surf B Biointerfaces. 2014, 114, 20-27. DOI: 10.1016/j.colsurfb.2013.09.010.

[103] Mu, C. F.; Balakrishnan, P.; De Cui, F.; Yin, Y. M.; Lee, Y. B.; Choi, H. G.; Yong, C. S.; Chung, S. J.; Shim, C. K.; Kim, D. D. The Effects of Mixed MPEG-PLA/Pluronic ${ }^{\circledR} \quad$ Copolymer Micelles on the Bioavailability and Multidrug Resistance of Docetaxel. Biomaterials. 2010, 31, 2371-2379. DOI: 10.1016/j. biomaterials.2009.11.102.

[104] Song, H.; Geng, H.; Ruan, J.; Wang, K.; Bao, C.; Wang, J.; Peng, X.; Zhang, X.; Cui, D. Development of Polysorbate 80/Phospholipid Mixed Micellar Formation for Docetaxel and Assessment of its in Vivo Distribution in Animal Models. Nanoscale Res. Lett. 2011, 6 (354), 1-12. DOI: 10.1186/1556-276X-6-354.

[105] Xu, Z.; Chen, L.; Gu, W.; Gao, Y.; Lin, L.; Zhang, Z.; Xi, Y.; Li, Y The Performance of Docetaxel-Loaded Solid Lipid Nanoparticles Targeted to Hepatocellular Carcinoma. Biomaterials. 2009, 30, 226-232. DOI: 10.1016/j.biomaterials.2008.09.014.

[106] Feng, L.; Wu, H.; Ma, P.; Mumper, R. J.; Benhabbour, S. R. Development and Optimization of Oil-Filled Lipid Nanoparticles Containing Docetaxel Conjugates Designed to Control the Drug Release Rate in Vitro and in Vivo. Int. J. Nanomedicine. 2011, 6, 25452556. DOI: $10.2147 / \mathrm{IJN} . S 24954$.

[107] Muthu, M. S.; Kulkarni, S. A.; Raju, A.; Feng, S. S. Theranostic Liposomes of TPGS Coating for Targeted Co-Delivery of Docetaxel and Quantum Dots. Biomaterials. 2012, 33, 3494-3501. DOI: 10.1016/j. biomaterials.2012.01.036.

[108] Yuan, Z.; Chen, D.; Zhang, S.; Zheng, Z. Preparation, Characterization and Evaluation of Docetaxel-Loaded, Folate-Conjugated PEGLiposomes. Yakugaku Zasshi. 2010, 130 (10), 1353-1359. DOI: 10.1248/yakushi.130.1353.

[109] Ling, Y.; Wei, K.; Luo, Y.; Gao, X.; Zhong, S. Dual Docetaxel/Superparamagnetic Iron Oxide Loaded Nanoparticles for Both Targeting Magnetic Resonance Imaging and Cancer Therapy. Biomaterials. 2011, 32, 7139-7150. DOI: 10.1016/j.biomaterials.2011.05.089.

[110] Liu, Y.; Li, K.; Pan, J.; Liu, B.; Feng, S. S. Folic Acid Conjugated Nanoparticles of Mixed Lipid Monolayer Shell and Biodegradable Polymer Core for Targeted Delivery of Docetaxel. Biomaterials. 2010, 31, 330-338. DOI: 10.1016/j.biomaterials.2009.09.036.

[111] Mi, Y.; Zhao, J.; Feng, S. S. Targeted Co-Delivery of Docetaxel, Cisplatin and Herceptin by Vitamin E TPGS-Cisplatin Prodrug Nanoparticles for Multimodality Treatment of Cancer. J. Control. Release. 2013, 169, 185-192. DOI: 10.1016/j.jconrel.2013.01.035.

[112] Lian, H.; Sun, J.; Zhang, T. A Rapid and Sensitive Determination of Paclitaxel in Rat Plasma by UPLC-MS/MS Method: Application to a Pharmacokinetic Study. Asian J. Pharm. Sci. 2013, 8, 199-205. DOI: 10.1016/j.ajps.2013.07.026.

[113] Liu, B.; Gou, X.; Bai, X.; Hou, X.; Li, D.; Zhong, G.; Jin, J.; Huang, M. Simultaneous Determination of Seven Taxoids in Rat Plasma by Uplc-Ms/Ms and Pharmacokinetic Study After Oral Administration of Taxus Yunnanensis Extracts. J. Pharm. Biomed. Anal. 2015, 107, 346-354. DOI: 10.1016/j.jpba.2015.01.001.

[114] Zhao, X.; Bi, K.; Wang, X.; Xue, X.; He, B.; Cui, Y.; Liu, Z.; Wang, D.; Chen, X. A UFLC-MS/MS Method Coupled with One-Step Protein Precipitation for Determination of Docetaxel in Rat Plasma: Comparative Pharmacokinetic Study of Modified Nanostructured Lipid Carrier. J. Pharm. Biomed. Anal. 2013, 83, 202-208. DOI: 10.1016/j. jpba.2013.05.025.

[115] Liu, Z.; Zhang, B.; Liu, Z.; Li, S.; Li, G.; Geng, L.; Zhao, X.; Bi, K.; Tang, X.; Chen, X. Development of a UPLC-ESI-MS/MS Method for the Determination of Larotaxel in Beagle Dog Plasma: Application to the Pharmacokinetic Study. Anal. Bioanal. Chem. 2012, 403, 323-330. DOI: 10.1007/s00216-012-5763-7. 